(CASE SERIES)

\title{
Physiotherapy management of geriatric COVID-19 patients in an intensive care unit of a government tertiary care hospital: A case series
}

\author{
Dipti Baban Geete 1, *, , Jaimala Vijay Shetye 2,\# and Anil Manohar Sathe 3, \# \\ 1. Assistant Professor, Physiotherapy School \& Center, Seth. G.S. Medical College \& KEM Hospital, Parel, Mumbai-12. \\ ${ }^{2}$ Associate Professor, Physiotherapy School \& Center, Seth. G.S. Medical College \& KEM Hospital, Parel, Mumbai-12. \\ ${ }^{3}$ Post-Graduate Student, Physiotherapy School \& Center, Seth. G.S. Medical College \& KEM Hospital, Parel, Mumbai-12 \\ \# All three authors contributed equally to the work.
}

Publication history: Received on 18 November 2020; revised on 25 November 2020; accepted on 28 November 2020

Article DOI: https://doi.org/10.30574/wjarr.2020.8.3.0447

\begin{abstract}
COVID-19 caused by SARS-CoV-2 has resulted in an alarming rate of mortality and morbidity worldwide, particularly in older population. It began as an outbreak in December 2019. The contribution of physiotherapy in preventing complications of restricted mobility and early recovery has been aptly acknowledged by the medical fraternity. This paper aims to share our experience and strategies as front liner Physiotherapists in treating critically ill geriatric patients with COVID-19, admitted in the Intensive Care Unit through case series of 3 patients.
\end{abstract}

Key-words: COVID-19; Physiotherapy; Comorbidities; Intensive Care Unit.

\section{Introduction}

The global outbreak of Severe Acute Respiratory Syndrome coronavirus 2 (SARS-CoV-2) disease 2019 (COVID-19) is having a dramatic effect throughout the world. This case series reflects the physiotherapy approach in geriatric rehabilitation associated with COVID-19 infection. SARS-CoV-2 is a novel disease that had emerged in December 2019 in China which spread rapidly all over the world including India. The first case of COVID-19 in India was reported in January 2020. Since it is novel, and the causative agent of a potentially fatal disease that is of great global public health concern, hence, the literature related to COVID-19 and guidelines regarding its modes of transmission, safety measures, intervention, etc. are constantly being updated as more and more knowledge is being shared all over [1].

In the COVID-19 pandemic, the older adults have been categorized as high risk. The reason being presence of other comorbidities like diabetes, hypertension, ischemic heart disease, and others that may be associated with aging. Clinical observation in this population highlights respiratory impairment, vascular consequences, effect of bed rest, effects of polypharmacy, and aggravation of frail state caused by COVID-19 infection. With advancing age, there is a decline in the physiological functions of the body as a whole [2]. The mortality and morbidity are found to be higher in this age group. According to Korea disease control, the fatality rate was high in the age group of more than 60 years of age, reaching $16.9 \%$, and $24.4 \%$ in the age group of $70-79$ years and $>80$ years respectively $[3,4]$.

SARS-Co-2 has been established as a cause of severe alveolar damage and pneumonia in patients with COVID-19. The consolidation of lung parenchyma precipitates the alterations in blood gases resulting in hypoxemic respiratory failure. There is involvement of not only the lungs but multiple organ systems like the central nervous system. As one of the functions of the central nervous system is to regulate the breathing process, it is a daunting task to compute the extent to which the failure of the central regulation of the breathing process contributes to the mortality in these patients.

\footnotetext{
${ }^{*}$ Corresponding author: Dipti Geete

Dipti B Geete, Assistant Professor, Physiotherapy School \& Center, Seth. G.S. Medical College \& KEM Hospital, Parel, Mumbai-12. 
At the level of alveolar capillary membrane, SARS-CoV-2 causes exudative and organized diffuse alveolar damage. The ongoing exudation along with fibrosis in the terminal bronchioles and alveolar walls thicken the gaseous exchange barrier further leading to profound hypoxia, threatening hypoxemic-respiratory failure. It has been observed that COVID patients may show normal saturation at rest but may de-saturate on doing any activity. It is also seen that some patients with a severe drop in PO2 remain asymptomatic initially due to a possible compensation by an increase in the rate of breathing that comes into play by the neurogenic mechanism to combat hypoxia resulting from a poor diffusion of 02 across the alveolar barrier. A possible explanation for such severe hypoxemia occurring in a compliant lung is the possible loss of lung perfusion and hypoxic vasoconstriction [5, 6].

Physiotherapeutic interventions play an important role as a complementary intervention in geriatric patients to treat and improve the overall functional level and to optimizing patient's recovery, as it takes into consideration the severity of respiratory symptoms and prevents further respiratory deterioration preserving of existing functional capabilities. This is because geriatric subjects have issues like fear of fall, balance impairments, osteoarthritis, which make independent mobility difficult $[7,8]$.

Physiotherapy is an integral part of the ICU team supporting the holistic treatment of patients with non-invasive, nonpharmacological treatment modalities mainly involving body positioning, breathing exercises, limb mobilization, relaxation, functional training of patients, and assisting in ventilator support weaning trials [9].

The choice of physiotherapy intervention and its intensity is individually tailored based on patient's health condition, comorbidity, and the respiratory potential. The safety of the patients as well as the physiotherapist is important more so because physiotherapy interventions are usually aerosol generating. Personal protective Equipment acts as a barrier while giving physiotherapy. The verbal instructions are not clearly audible through the mask and face shield especially in the elderly with hearing impairment. Contactless Physiotherapy as laid in the guideline is also not practical in the elderly due to fear of losing balance. We present here three elderly cases, successfully treated overcoming these challenges while delivering Physiotherapy.

\section{Case Study - 01}

A 70-year-old female was admitted to ICU on the 3rd day of onset of symptoms (fever with, dry cough, severe breathlessness, myalgia, vomiting, loss of smell and dizziness) and had type II b hypoxia. She had associated comorbidities of ischemic heart disease and hypertension (15 years). Her saturation was maintained on 15 liters of oxygen with non-rebreathing mask ventilation. HRCT was suggestive of atypical pneumonia (CORADS 6). 50 to 60\% area of lung involvement was reported with bilateral ground glass opacities forming consolidation, interlobular septal thickening in both the lung fields predominantly in sub pleural location. Increased cardiothoracic ratio suggested cardiomegaly. The first session of physiotherapy began with patient education followed by breathing control and thoracic mobility exercises in the supine position, sitting. The session ended with prone positioning which she maintained for 30 to 45 minutes as tolerated. Exercises of the upper and lower limbs were included as the condition stabilized. While performing in bed and bedside mobility exercises, she was hemodynamically stable; as assessed by pulse rate ECG and oxygen saturation as noted from the monitors. She was further progressed to performing exercises in standing like Spot Marching, walking around the bed with oxygen support and later walking in ICU with oxygen tube extension. Even though the patient falls under the geriatric age group, she could tolerate the exercises well and made a quick recovery. Sessions were concluded with relaxation in a prone position. (Table 1)

\section{Case Study - 02}

A 70-year old male was hospitalized for a COVID-19 infection on the 2nd day of onset of symptoms (fever with chills, dry cough, severe breathlessness, myalgia, and headache) and transferred to ICU on the same day with type II b hypoxia. He had associated comorbidities of hypertension (5 years), ischemic heart disease. He was on NIV, Fio $270 \%$ and PEEP $8 \mathrm{~cm}$ of $\mathrm{H}_{2} \mathrm{O}$. He would de-saturate up to $88 \% \mathrm{Spo} 2$ with exercise and hence his oxygen support was increased by 2 liters before starting the exercises. He benefited from crocodile breathing exercise i.e. posterior basal lung expansion exercise in prone position as evidenced by an improvement in $\mathrm{SPO}_{2}$. Exercises were gradually progressed from in bed mobility exercises to out of bed mobility exercises as well as an exercise in standing keeping in consideration the hemodynamic stability of the patient. Sessions were concluded by relaxation in the prone position. (Table 2) 


\section{Case study- 03}

A 60 year old male, was hospitalized in a private hospital for 18 days, due to financial constraint he came to the public sector ICU. Initially, he was on NIV and maintained oxygen saturation for 2 days. He was symptomatic (fever with, dry cough, severe breathlessness, loss of smell, vomiting, and fatigue) with per rectal bleeding. He had associated comorbidities of diabetes (11 years), hypertension ( 5 years) and ischemic heart disease. CT Pulmonary angiogram done on the 4th day of admission suggested asymmetric bilateral diffuse ground glass opacities intermixed with few septal thickening, more marked in the lower lobes. Mild abnormal dilation of the central as well as peripheral bronchioles was seen. Few tiny parenchymal cysts were seen in the anterior as well as the posterior segment of the right upper lobe. Similar findings were noted by HRCT at the time of discharge from the private hospital. Physiotherapy session began with patient education followed by breathing control and thoracic mobility exercises in the supine position, sitting further progressed to standing, spot marching, and walking with adequate rest pauses with respect to hemodynamic stability. Sessions were concluded by relaxation in prone body position. (Table 3)

Table 1 Physiotherapeutic intervention with hemodynamic details- Case 01

\begin{tabular}{|c|c|c|c|c|c|c|c|c|c|}
\hline \multicolumn{2}{|c|}{ Days of PT treatment } & 1 & 2 & 3 & 4 & 5 & 6 & 7 & 8 \\
\hline \multicolumn{2}{|l|}{ Mode } & NRBM & NRBM & NRBM & NRBM & FM & FM & FM & NP \\
\hline \multicolumn{2}{|c|}{ Oxygen support (L/min) } & $15 / \mathrm{L}$ & $10 / \mathrm{L}$ & $12 / \mathrm{L}$ & $8 / \mathrm{L}$ & $5 / \mathrm{L}$ & $8 / \mathrm{L}$ & $5 / \mathrm{L}$ & $2 / \mathrm{L}$ \\
\hline \multirow[t]{2}{*}{ Pulse(bpm) } & Pre & 76 & 76 & 69 & 68 & 88 & 48 & 56 & 66 \\
\hline & Post & $x$ & 89 & 63 & 74 & 93 & 51 & 63 & 74 \\
\hline \multirow[t]{2}{*}{ Spo2 (\%) } & Pre & 94 & 94 & 84 & 98 & 93 & 93 & 96 & 94 \\
\hline & post & $x$ & 96 & 95 & 97 & 96 & 93 & 96 & 97 \\
\hline \multicolumn{2}{|l|}{ Positioning } & $x$ & $\sqrt{ }$ & $\sqrt{ }$ & $\sqrt{ }$ & $\sqrt{ }$ & $\sqrt{ }$ & $\sqrt{ }$ & $\sqrt{ }$ \\
\hline \multicolumn{2}{|c|}{ Breathing exercises } & $x$ & $\sqrt{ }$ & $\sqrt{ }$ & $\sqrt{ }$ & $\sqrt{ }$ & $\sqrt{ }$ & $\sqrt{ }$ & $\sqrt{ }$ \\
\hline \multicolumn{2}{|c|}{ In bed mobility } & $x$ & $x$ & $x$ & $\sqrt{ }$ & $\sqrt{ }$ & $\sqrt{ }$ & $\sqrt{ }$ & $\sqrt{ }$ \\
\hline \multicolumn{2}{|c|}{ Sitting exercises } & $x$ & $x$ & $x$ & $x$ & $\sqrt{ }$ & $x$ & $\sqrt{ }$ & $\sqrt{ }$ \\
\hline \multicolumn{2}{|l|}{ Standing } & $x$ & $x$ & $x$ & $x$ & $x$ & $x$ & $\sqrt{ }$ & $\sqrt{ }$ \\
\hline \multicolumn{2}{|c|}{ Spot marching } & $x$ & $x$ & $x$ & $x$ & $x$ & $x$ & $x$ & $\sqrt{ }$ \\
\hline \multicolumn{2}{|l|}{ walking } & $x$ & $x$ & $x$ & $x$ & $x$ & $x$ & $x$ & $\sqrt{ }$ \\
\hline
\end{tabular}

\begin{tabular}{|c|c|c|c|c|c|c|c|c|c|}
\hline \multicolumn{2}{|c|}{ Days of PT treatment } & 9 & 10 & 11 & 12 & 13 & 14 & 15 & 16 \\
\hline \multicolumn{2}{|l|}{ Mode } & NP & $\mathrm{NP}$ & NP & NP & NP & NP & RA & RA \\
\hline \multicolumn{2}{|c|}{ Oxygen support (L/min) } & $4 / \mathrm{L}$ & $2 / \mathrm{L}$ & $4 / \mathrm{L}$ & $2 / \mathrm{L}$ & $2 / \mathrm{L}$ & $1 / \mathrm{L}$ & Off 02 & Off 02 \\
\hline \multirow[t]{2}{*}{ Pulse(bpm) } & Pre & 70 & 75 & 77 & 72 & 72 & 82 & 76 & 88 \\
\hline & Post & 83 & 89 & 79 & 92 & 84 & 94 & 88 & 100 \\
\hline \multirow[t]{2}{*}{ Spo2 (\%) } & Pre & 98 & 93 & 92 & 95 & 99 & 96 & 97 & 98 \\
\hline & post & 96 & 95 & 94 & 95 & 98 & 98 & 95 & 100 \\
\hline \multicolumn{2}{|l|}{ Positioning } & $\sqrt{ }$ & $\sqrt{ }$ & $\sqrt{ }$ & $\sqrt{ }$ & $\sqrt{ }$ & $\sqrt{ }$ & $\sqrt{ }$ & $\sqrt{ }$ \\
\hline \multicolumn{2}{|c|}{ Breathing exercises } & $\sqrt{ }$ & $\sqrt{ }$ & $\sqrt{ }$ & $\sqrt{ }$ & $\sqrt{ }$ & $\sqrt{ }$ & $\sqrt{ }$ & $\sqrt{ }$ \\
\hline \multicolumn{2}{|c|}{ In bed mobility } & $\sqrt{ }$ & $\sqrt{ }$ & $\sqrt{ }$ & $\sqrt{ }$ & $\sqrt{ }$ & $\sqrt{ }$ & $\sqrt{ }$ & $\sqrt{ }$ \\
\hline \multicolumn{2}{|c|}{ Sitting exercises } & $\sqrt{ }$ & $\sqrt{ }$ & $\sqrt{ }$ & $\sqrt{ }$ & $\sqrt{ }$ & $\sqrt{ }$ & $\sqrt{ }$ & $\sqrt{ }$ \\
\hline \multicolumn{2}{|l|}{ Standing } & $x$ & $\sqrt{ }$ & $\sqrt{ }$ & $\sqrt{ }$ & $\sqrt{ }$ & $\sqrt{ }$ & $\sqrt{ }$ & $\sqrt{ }$ \\
\hline \multicolumn{2}{|c|}{ Spot marching } & $x$ & $\sqrt{ }$ & $\sqrt{ }$ & $\sqrt{ }$ & $\sqrt{ }$ & $\sqrt{ }$ & $\sqrt{ }$ & $\sqrt{ }$ \\
\hline \multicolumn{2}{|l|}{ walking } & $x$ & $\sqrt{ }$ & $\sqrt{ }$ & $\sqrt{ }$ & $\sqrt{ }$ & $\sqrt{ }$ & $\sqrt{ }$ & $\sqrt{ }$ \\
\hline \multicolumn{2}{|l|}{ BHT } & \multicolumn{8}{|c|}{$\begin{array}{l}\text { Date: } 28 / 8 / 20 \text {, Oxygen support: } 2 \mathrm{~L} / \mathrm{min} \text {, Count } 4 \\
\text { Date: } 5 / 9 / 20 \text {, Oxygen support: off } 02 \text {, Count } 30\end{array}$} \\
\hline
\end{tabular}


Table 2 Physiotherapeutic intervention with hemodynamic details- Case 02

\begin{tabular}{|c|c|c|c|c|c|c|c|c|c|c|c|}
\hline \multicolumn{2}{|c|}{ Days of PT treatment } & 1 & 2 & 3 & 4 & 5 & 6 & 7 & 8 & 9 & 10 \\
\hline \multicolumn{2}{|l|}{ Mode } & NIV & NRBM & NRBM & NRBM & NRBM & NEBM & NRBM & $\mathrm{FM}$ & NP & NP \\
\hline \multicolumn{2}{|l|}{$\begin{array}{l}\text { Oxygen } \\
\text { (L/min) }\end{array}$} & $\begin{array}{l}\text { Fio2 } 70 \% \text { PEEP } \\
8\end{array}$ & $13 / \mathrm{L}$ & $15 / \mathrm{L}$ & $10 / \mathrm{L}$ & $8 / \mathrm{L}$ & $8 / \mathrm{L}$ & $6 / L$ & $10 / \mathrm{L}$ & 6/L & $2 / \mathrm{L}$ \\
\hline \multirow[t]{2}{*}{ Pulse(bpm) } & Pre & 70 & 62 & 72 & 57 & 84 & 82 & 69 & 76 & 76 & 83 \\
\hline & Post & 72 & 70 & 77 & 69 & 101 & 90 & 87 & 100 & 88 & 79 \\
\hline \multirow[t]{2}{*}{ Spo2 (\%) } & Pre & 95 & 92 & 94 & 86 & 88 & 96 & 91 & 97 & 96 & 97 \\
\hline & post & 06 & 97 & 99 & 97 & 99 & 100 & 99 & 100 & 98 & 98 \\
\hline \multicolumn{2}{|l|}{ Positioning } & $\sqrt{ }$ & $\sqrt{ }$ & $\sqrt{ }$ & $\sqrt{ }$ & $\sqrt{ }$ & $\sqrt{ }$ & $\sqrt{ }$ & $\sqrt{ }$ & $\sqrt{ }$ & $\sqrt{ }$ \\
\hline \multicolumn{2}{|c|}{ Breathing exercises } & $\sqrt{ }$ & $\sqrt{ }$ & $\sqrt{ }$ & $\sqrt{ }$ & $\sqrt{ }$ & $\sqrt{ }$ & $\sqrt{ }$ & $\sqrt{ }$ & $\sqrt{ }$ & $\sqrt{ }$ \\
\hline \multicolumn{2}{|c|}{ In bed mobility } & $\times$ & $\times$ & $\sqrt{ }$ & $\sqrt{ }$ & $\sqrt{ }$ & $\sqrt{ }$ & $\sqrt{ }$ & $\sqrt{ }$ & $\sqrt{ }$ & $\sqrt{ }$ \\
\hline \multicolumn{2}{|c|}{ Sitting exercises } & $x$ & $x$ & $\sqrt{ }$ & $\sqrt{ }$ & $\sqrt{ }$ & $\sqrt{ }$ & $\sqrt{ }$ & $\sqrt{ }$ & $\sqrt{ }$ & $\sqrt{ }$ \\
\hline \multicolumn{2}{|l|}{ Standing } & $x$ & $\times$ & $\times$ & $\times$ & $\times$ & $\sqrt{ }$ & $\times$ & $\sqrt{ }$ & $\sqrt{ }$ & $\sqrt{ }$ \\
\hline \multicolumn{2}{|c|}{ Spot marching } & $x$ & $x$ & $x$ & $x$ & $x$ & $x$ & $x$ & $\sqrt{ }$ & $\sqrt{ }$ & $\sqrt{ }$ \\
\hline \multicolumn{2}{|l|}{ walking } & $x$ & $x$ & $x$ & $x$ & $x$ & $x$ & $x$ & $\sqrt{ }$ & $\sqrt{ }$ & $\sqrt{ }$ \\
\hline \multicolumn{2}{|l|}{ BHT } & \multicolumn{10}{|c|}{ Date: $28 / 8 / 20$, Oxygen support: $13 \mathrm{~L} / \mathrm{min}$, Count 7} \\
\hline
\end{tabular}

Table 3 Physiotherapeutic intervention with hemodynamic details- Case 03

\begin{tabular}{|c|c|c|c|c|c|c|c|c|c|}
\hline \multicolumn{2}{|c|}{ Days of PT treatment } & 1 & 2 & 3 & 4 & 5 & 6 & 7 & 8 \\
\hline \multicolumn{2}{|l|}{ Mode } & NIV & NRBM & NRBM & NRBM & NRBM & FM & NP & NP \\
\hline \multicolumn{2}{|c|}{ Oxygen support (L/min) } & $x$ & $8 / \mathrm{L}$ & $15 / \mathrm{L}$ & $8 / \mathrm{L}$ & $9 / \mathrm{L}$ & $6 / \mathrm{L}$ & $4 / \mathrm{L}$ & $1 / \mathrm{L}$ \\
\hline \multirow[t]{2}{*}{ Pulse(bpm) } & Pre & $\times$ & 103 & 99 & 91 & 94 & 96 & 105 & 111 \\
\hline & Post & $x$ & 88 & 89 & 79 & 85 & 83 & 84 & 109 \\
\hline \multirow[t]{2}{*}{ Spo2 (\%) } & Pre & $x$ & 94 & 96 & 100 & 96 & 91 & 91 & 99 \\
\hline & post & $x$ & 95 & 97 & 100 & 95 & 94 & 96 & 98 \\
\hline \multicolumn{2}{|l|}{ Positioning } & $x$ & $\sqrt{ }$ & $\sqrt{ }$ & $\sqrt{ }$ & $\sqrt{ }$ & $\sqrt{ }$ & $\sqrt{ }$ & $\sqrt{ }$ \\
\hline \multicolumn{2}{|c|}{ Breathing exercises } & $x$ & $\sqrt{ }$ & $\sqrt{ }$ & $\sqrt{ }$ & $\sqrt{ }$ & $\sqrt{ }$ & $\sqrt{ }$ & $\sqrt{ }$ \\
\hline \multicolumn{2}{|c|}{ In bed mobility } & $x$ & $\sqrt{ }$ & $\sqrt{ }$ & $\sqrt{ }$ & $\sqrt{ }$ & $\sqrt{ }$ & $\sqrt{ }$ & $\sqrt{ }$ \\
\hline \multicolumn{2}{|c|}{ Sitting exercises } & $x$ & $x$ & $x$ & $\sqrt{ }$ & $\sqrt{ }$ & $\sqrt{ }$ & $\sqrt{ }$ & $\sqrt{ }$ \\
\hline \multicolumn{2}{|l|}{ Standing } & $x$ & $x$ & $x$ & $x$ & $\sqrt{ }$ & $\sqrt{ }$ & $\sqrt{ }$ & $\sqrt{ }$ \\
\hline \multicolumn{2}{|c|}{ Spot marching } & $x$ & $x$ & $x$ & $x$ & $x$ & $x$ & $\sqrt{ }$ & $\sqrt{ }$ \\
\hline \multicolumn{2}{|l|}{ walking } & $\times$ & $x$ & $\times$ & $x$ & $\times$ & $x$ & $x$ & $\sqrt{ }$ \\
\hline \multicolumn{2}{|l|}{ BHT } & \multicolumn{8}{|c|}{$\begin{array}{l}\text { Date: } 28 / 8 / 20 \text {, Oxygen support: } 8 \mathrm{~L} / \mathrm{min} \text {, Count } 6 \\
\text { Date: } 5 / 9 / 20 \text {, Oxygen support: } 1 \mathrm{~L} / \mathrm{min} \text {, Count }\end{array}$} \\
\hline
\end{tabular}

NIV: Non Invasive Ventilation; NRBM: Non rebreathing mask; NP: Nasal prongs; $\mathrm{O}_{2}$ : Oxygen; $\mathrm{Po}_{2}$ FiO2: Fraction of Inspired Oxygen;

L/min: Liters/min; FM: face mask; BHT: Breath holding time; PEEP: positive end-expiratory pressure; RA: room air. 


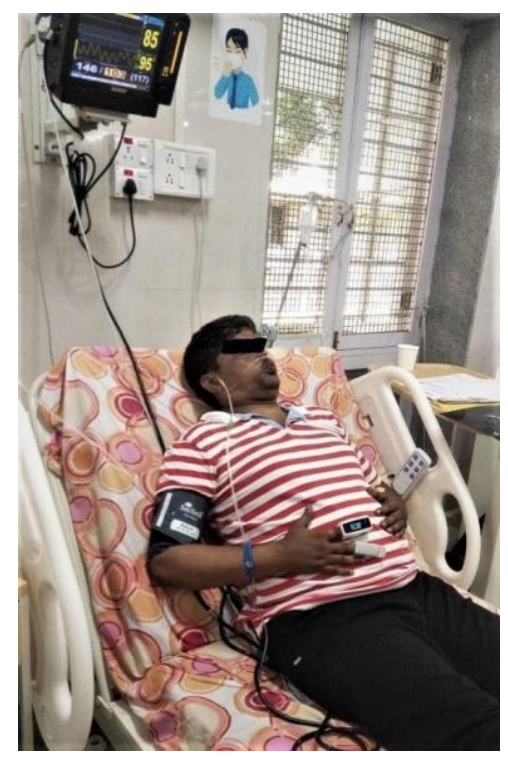

Figure 1 Exercises in propped up position

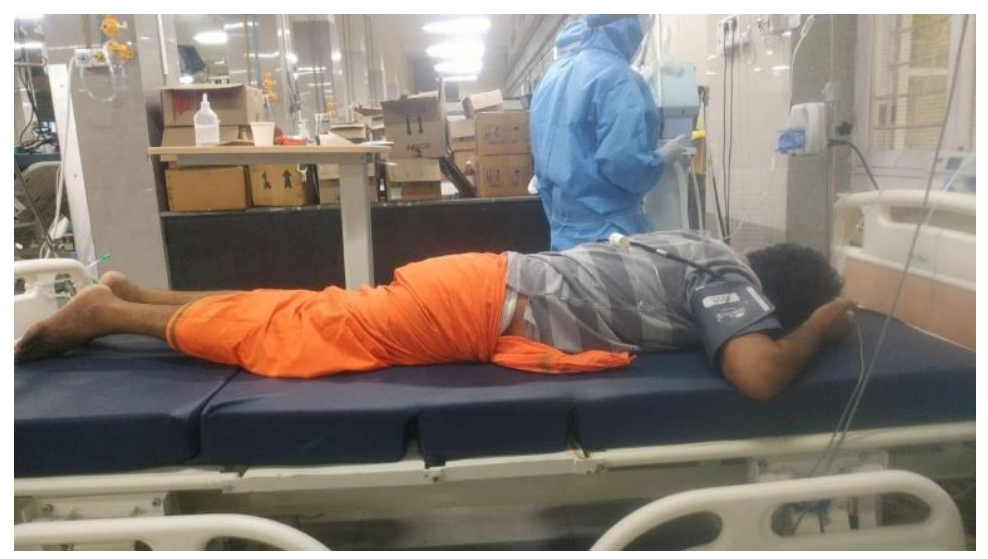

Figure 2 Crocodile breathing exercise in prone

\section{Discussion}

Elderly patients with comorbidities are more sensitive to coronavirus disease. They have prolonged ICU stay which may result in ICU acquired weakness and also show psychological impact. Wei-Jie Guan et al have reported that poorer clinical outcomes in patients with increased comorbidity than those without a greater number of comorbidities correlated with poorer clinical outcomes $[10,11]$. Hence, acute care Physiotherapy is indicated immediately after the patient is hemodynamically stable. Patients in ICU were on various ventilatory supports. Initially in the ICU, the physiotherapy goal was to improve lung function and reduce complications arising due to immobility. Patients had difficulty to accept various ventilatory modes like NIV, Non ReBreathing Mask, nasal prongs, Face mask, and this posed difficulty in assuming the prone position [12].

This was overcome by educating the patient regarding ventilatory support and guiding about his/her health condition. This essentially developed a rapport with the patient. COVID-19 pneumonia primarily affects the lower lobes and hence physiotherapy intervention like crocodile and diaphragmatic breathing along with prone positioning facilitates lung expansion and improves oxygen saturation.

Physiotherapy intervention began with patient education about the problem and the need for pulmonary rehabilitation. Patients were instructed and given body position changes every 30 minutes to 2 hours (prone, lying on the right side, sitting upright 60-90 degrees and lying on the left side) as per the CARP protocol to improve hypoxemia [13]. Patients 
were given diaphragmatic breathing exercises prior to general mobility exercises. The therapy session concluded with crocodile breathing exercises. Adequate rest pauses were given during and after each set of exercises. A total of 30 repetitions of both the breathing exercises were done daily under supervision. This whole routine was done once per day. Graded mobility exercises were given to prevent peripheral deconditioning and bed rest, ankle toe movement, heel slides were given as in bed mobility exercises. Exercises were further progressed to sitting at the edge of the bed, standing, spot marching and walking in the ICU with adequate rest-pause followed by positioning [14, 15]. Walking program in the ICU with an extended tube despite of Non-invasive ventilatory support helped to increase the intensity of mobilization exercises to improve oxygen perfusion. Safe multi-model physiotherapy as per the individual need of the elderly giving due consideration to hemodynamic response helped patients to wean from oxygen support [16].

Patients were given diaphragmatic breathing exercises and crocodile breathing exercises which played an important role in improving oxygen saturation of patients and weaning from NIV mode. It also helped to reduce the respiratory rate and hence the work of breathing. For performing Crocodile breathing exercises, patients were instructed to lie on the stomach with their hands in a diamond shape supporting their forehead. In presence of the mask of NIV, the patients were allowed to rotate their head to one side to accommodate the mask as seen in the figure 2 . They were cued to try and push their ribs out laterally and try to breathe all the way down to the sacrum [17]. This results in the ventilation of posterior basal areas of the lung which are likely to undergo atelectasis if the patients remain in the supine position all day. The ground glass opacities are also commonly found in the basal areas of the lungs. Hence crocodile breathing has an added advantage of prone position, which has been evidenced to improve oxygenation. Similar to diaphragmatic breathing exercise, crocodile breathing also recruits diaphragm muscle. The breath holding time also improved in all the three patients as seen in the tables.

The above patients were attended on one to one basis by the same Physiotherapist daily. The respiratory care, graded mobilization, walking program with supportive care along with psychological support played an essential role in all these patients towards weaning from supplemental oxygen support. All three patients were discharged, without the need for home oxygen support. Positive outcomes were obtained with physiotherapy interventions in all the three patients despite their diverse characteristics.

\section{Conclusion}

An individually tailored Physiotherapy regimen helps elderly COVID 19 patients towards a successful recovery. The case series highlights the patient's difficulty to adapt to the various ventilatory modes and the physiotherapist's difficulty in communicating with the patient in PPE and in planning and implementing a safe yet effective exercise program due to the presence of comorbidities like diabetes, hypertension and ischemic heart disease. The decision making is on daily basis considering the hemodynamically unstable state of the patients in ICU. Reading the previous day's notes help in this decision making and planning of the daily schedule. Hence we conclude that physiotherapy intervention was well tolerated by the patients and they were able to return to the community.

\section{Abbreviations}

COVID-19: Coronavirus disease; SARS-CoV-2: severe acute respiratory syndrome coronavirus 2; ICU: Intensive Care Unit; NIV: Non Invasive Ventilation; NRBM; Non rebreathing mask; NP: Nasal prongs; ARDS: Acute respiratory distress syndrome; CARP: COVID Awake Repositioning Protocol; $\mathrm{O}_{2}$ : Oxygen; $\mathrm{Po}_{2}$ :Partial pressure of oxygen; Fi02: Fraction of Inspired Oxygen; L/min: Liters/minute; FM: face mask; BHT: Breath holding time.

\section{Compliance with ethical standards}

\section{Acknowledgments}

The authors acknowledge Dr. Hemant Deshmukh (Dean, Seth GSMC \& KEM Hospital Mumbai), Dr. Milind Nadkar (Academic Dean, Seth GSMC \& KEM Hospital). Professor Saraswati Iyer (Professor and head, Physiotherapy school and center, Seth GSMC and KEMH) , Dr. Sushama A Bhandare (Assistant Professor, Physiotherapy school and center, Seth GSMC and KEMH) , Dr. Mariya P Jiandani ( Associate Professor, Physiotherapy school and center, Seth GSMC and KEMH), Dr. Kaustubh Salagre (Associate Professor, General Medicine, GSMC and KEMH) and all Patients whose information was used. 


\section{Disclosure of conflict of interest}

None.

\section{Statement of informed consent}

Informed consent was obtained from all individual participants included in the study.

\section{References}

[1] Singhal T. A review of coronavirus disease-2019 (COVID-19). The Indian Journal of Pediatrics. 2020 Mar; 13:1-6.

[2] Kang SJ, Jung SI. Age-related morbidity and mortality among patients with COVID-19. Infection \& chemotherapy. 2020 Jun; 52(2):154.

[3] Komorowski, M., and Aberegg, S. K. 2020 Using applied lung physiology to understand COVID-19 patterns [published online ahead of print, 2020 May 27]. Br. J. Anaesth., DOI: 10.1016/j.bja.2020.05.019.

[4] Baig AM. Computing the Effects of SARS-CoV-2 on Respiration Regulatory Mechanisms in COVID-19. ACS Chemical Neuroscience. 2020 Jun 29; 11(16):2416-21.

[5] Millán-Oñate J, Millan W, Mendoza LA, Sánchez CG, Fernandez-Suarez H, Bonilla-Aldana DK, Rodríguez-Morales AJ. Successful recovery of COVID-19 pneumonia in a patient from Colombia after receiving chloroquine and clarithromycin. Annals of Clinical Microbiology and Antimicrobials. 2020 Dec; 19:1-9.

[6] Ronco JJ, Fenwick JC, Tweeddale MG, Wiggs BR, Phang PT, Cooper DJ, Cunningham KF, Russell JA, Walley KR, Chernow B, Dantzker D. Identification of the critical oxygen delivery for anaerobic metabolism in critically III septic and nonseptic humans. Jama. 1993 Oct 13; 270(14):1724-30.

[7] Vitacca M, Carone M, Clini EM, Paneroni M, Lazzeri M, Lanza A, Privitera E, Pasqua F, Gigliotti F, Castellana G, Banfi P. Joint statement on the role of respiratory rehabilitation in the COVID-19 crisis: the Italian position paper. Respiration. 2020:1-7.

[8] Kachpile ST, Lohakare PK, Jiandani MP et. al. Physiotherapy interventions in COVID-19 patient with multiple comorbidities: a case report. Int J Health Sci Res. 2020; 10(10):96-101.

[9] Verma CV, Arora RD, Shetye JV, Karnik ND, Patil PC, Mistry HM, Kubal SV, Kolwankar NS, Dalvi AA, Vichare SA, Desai UD. Guidelines of physiotherapy management in acute care of COVID-19 at dedicated COVID center in Mumbai. Physiotherapy-The Journal of Indian Association of Physiotherapists. 2020 Jan 1; 14(1):55.

[10] Guan WJ, Liang WH, Zhao Y, Liang HR, Chen ZS, Li YM, Liu XQ, Chen RC, Tang CL, Wang T, Ou CQ. Comorbidity and its impact on 1590 patients with Covid-19 in China: A Nationwide Analysis. European Respiratory Journal. 2020 May $1 ; 55(5)$.

[11] Hosey MM, Needham DM. Survivorship after COVID-19 ICU stay. Nature Reviews Disease Primers. 2020 Jul 15 ; 6(1):1-2.

[12] Simpson R, Robinson L. Rehabilitation After Critical Illness in People With COVID-19 Infection. American journal of physical medicine \& rehabilitation. 2020 Jun; 99(6):470.

[13] COVID Awake Repositioning Protocol; (CARP). Resuscitation \& Acute Critical Care, Janus General Medicine; 2020

[14] Sommers J, Engelbert RH, Dettling-Ihnenfeldt D, Gosselink R, Spronk PE, Nollet F, van der Schaaf M. Physiotherapy in the intensive care unit: an evidence-based, expert driven, practical statement and rehabilitation recommendations. Clinical rehabilitation. 2015 Nov; 29(11):1051-63.

[15] Yang LL, Yang T. Pulmonary rehabilitation for patients with coronavirus disease 2019 (COVID-19). Chronic Diseases and Translational Medicine. 2020 May 14.

[16] da Silva CM, do Nascimento Andrade A, Nepomuceno B, Xavier DS, Lima E, Gonzalez I, Esquivel MS, Novais MC, Magalhães P, da Silva Almeida R, Gomes VA. Evidence-based Physiotherapy and Functionality in Adult and Pediatric patients with COVID-19. Journal of Human Growth and Development. 2020 Apr 14; 30(1):148-55.

[17] Gaffar AF, Waghray S, Balne NK. Efficacy of Balance Scales in Fall Risk Identification in Elderly with Breathing Pattern Dysfunction-An Observational Study. International Journal of Health Sciences and Research. 2019; 9(2):101-7. 